\title{
Tendencias de la oferta laboral colombiana y su relación con los perfiles académicos de la educación superior
}

\author{
Gloria Amparo Orrego-Agudelo* \\ Luz Dary Vega-Hernández ${ }^{* *}$
}

Doctoranda en Educación, Universidad Central de Venezuela. Docente y líder del grupo de investigación "Centro de Investigaciones en Mercadeo y Publicidad (CIMEP)", Universidad de Santander (UDEs), Bucaramanga, Colombia. Correo electrónico

gloriaamparoorrego@gmail.com

* Profesional en Mercado y Publicidad. Joven investigadora de Colciencias 2014, Universidad de Santander (UDES), Bucaramanga, Colombia.

Correo electrónico:

luzdvegah@gmail.com

Recibido: 30 de octubre del 2015

Aprobado: 11 de enero del 2016

Cómo citar este artículo: Orrego-Agudelo, Gloria Amparo y Luz Dary Vega-Hernández.

"Tendencias de la oferta laboral colombiana y su relación con los perfiles académicos de la educación superior". Rastros Rostros 18.32 (2016): 29-42. Impreso. doi: http://dx.doi. org/10.16925/ra.v18i32.1321

\section{Resumen}

Introducción: este artículo muestra los resultados de la investigación “Tendencias de la oferta laboral colombiana de profesionales en Mercadeo y Publicidad", que buscó identificar las oportunidades de desempeño laboral de profesionales de Mercadeo y Publicidad. Metodología: el estudio es descriptivo y relaciona las competencias, los cargos y las funciones acordes con las necesidades, al igual que los avances en ciencia, tecnología y desarrollo pertinentes a estas disciplinas. Se inició describiendo los perfiles de egresados de 28 programas académicos de Mercadeo, Publicidad y afines, inscritos en la Asociación de Programas de Mercadeo; también los requerimientos de profesionales de 136 empresas de cinco ciudades de Colombia. Resultados: se pudo constatar que un profesional en Mercadeo y Publicidad debe estar dispuesto a gerenciar proyectos con una amplia visión comercial acorde con las tendencias del marketing, los cambios económicos y los tratados de libre comercio que orientan al mercado local y ponen a prueba estrategias de posicionamiento de marca y desarrollo de productos. Conclusiones: se concluye que los diseños curriculares de los programas ofertados en Colombia son muy cercanos a las tendencias laborales demandadas por el medio empresarial, de manera que cumplen con las expectativas de formación de los sectores económicos del país.

Palabras clave: competencia, laboral, mercadeo, profesionales, publicidad, tendencias. 


\title{
Trends in the Colombian labor market and their relationship with the academic profiles of higher education
}

\begin{abstract}
Introduction: This article presents the results of the research project "Trends in the Colombian labor market for marketing and advertising professionals," which sought to identify the job opportunities for marketing and advertising professionals. Methodology: The study is descriptive and lists the competencies, positions, and functions according to the needs-as well as advances in science, technology, and development-relevant to these disciplines. It began by describing the profiles of graduates of 28 academic programs in marketing, advertising, and related areas who are members of the Association of Marketing Programs; it additionally described the requirements of professionals from 136 companies in five cities in Colombia. Results: It was found that a marketing and advertising professional must be prepared to manage projects with a broad business vision in line with the marketing trends, economic changes, and free trade agreements that guide the local market, using a variety of brand positioning and product development strategies. Conclusions: It concluded that the curricular design of the programs offered in Colombia is very close to the labor trends demanded by the business sector, thus meeting the training expectations of the country's economic sectors.
\end{abstract}

Keywords: competition, labor, marketing, professionals, advertising, trends.

\section{Tendências da oferta de trabalho colombiana e sua relação com os perfis acadêmicos da educação superior}

\section{Resumo}

Introdução: este artigo mostra os resultados da pesquisa "Tendências da oferta de trabalho colombiana de profissionais em marketing e publicidade", que procurou identificar as oportunidades de desempenho no trabalho de profissionais de marketing e publicidade. Metodologia: o estudo é descritivo e relaciona as competências, os cargos e as funções de acordo com as necessidades, bem como os avanços em ciência, tecnologia e desenvolvimento pertinentes a essas disciplinas. Começa-se descrevendo os perfis de alunos egressos de 28 programas acadêmicos de marketing, publicidade e afins, inscritos na Associação de Programas de Marketing; também as exigências de profissionais de 136 empresas de cinco cidades da Colômbia. Resultados: pode-se constatar que um profissional em marketing e publicidade deve estar disposto a gerenciar projetos com uma ampla visão comercial de acordo com as tendências do marketing, as mudanças econômicas e os tratados de livre comércio que orientam o mercado local e colocam à prova estratégias de posicionamento de marca e desenvolvimento de produtos. Conclusões: conclui-se que os desenhos curriculares dos programas ofertados na Colômbia são muito próximos às tendências de trabalho exigidas pelo meio empresarial, de maneira que satisfaz as expectativas de formação dos setores econômicos do país.

Palavras-chave: competência, trabalho, marketing, profissionais, publicidade, tendências. 


\section{Introducción}

El artículo presenta los resultados de la investigación "Tendencias de la oferta laboral colombiana de profesionales en Mercadeo y Publicidad", que hizo parte de la línea de investigación "Información y Comunicación Estratégica del Consumidor” del programa de Mercadeo y Publicidad de la Universidad de Santander (UdEs), Bucaramanga. Este estudio tuvo como propósito responder a la pregunta: ¿cuáles son las nuevas oportunidades de desempeño laboral de los profesionales de Mercadeo y Publicidad? Para esto se tuvo en cuenta las tendencias científicas y tecnológicas de estas disciplinas y las necesidades empresariales del medio en un entorno global. Responder a esta pregunta de investigación les permite a los programas que ofrecen estas disciplinas verificar el nivel de pertinencia del proceso de formación en relación con las exigencias y demandas del área laboral correspondiente. De esta forma, se busca un mayor posicionamiento en el medio empresarial local, regional e internacional.

Teniendo en cuenta lo anterior, el Centro de Investigaciones de Mercadeo y Publicidad de la udes, sede Bucaramanga, y la Asociación de Programas de Mercadeo (Aspromer) se unieron para trabajar conjuntamente en esta propuesta y obtener información confiable que sirviera en las reflexiones sobre el quehacer de los profesionales que actualmente se forman en las mencionadas áreas y las posibilidades de empleo que el medio empresarial tiene dispuestas para ellos. La metodología utilizada para la realización del trabajo de investigación fue descriptiva con un enfoque cuantitativo, que se vale de técnicas de medición y de análisis para las variables identificadas tanto en las competencias de desempeño laboral como las de los campos disciplinares. Las fuentes de información secundaria fueron proporcionadas por los programas académicos, y la información primaria fue ofrecida por las empresas objeto de estudio. Tanto la población como la muestra se conformaron y seleccionaron a partir de las grandes y medianas empresas de todos los sectores económicos, además de, como se expresó, 28 programas de Mercadeo y áreas afines que existen en el país, inscritos en Aspromer hacia febrero del 2014. Estos programas curriculares aportaron últimos la información documental sobre los perfiles profesionales. La muestra de las grandes y medianas empresas en que se basó el estudio debía estar inscrita en la Cámara de Comercio de las cinco ciudades principales de Colombia (Bogotá,
Medellín, Cali, Barranquilla y Bucaramanga). El instrumento para la recolección de esta información fue una encuesta, que permitió establecer las necesidades empresariales en referencia al desempeño laboral de los profesionales en Mercadeo y Publicidad; esta fue aplicada a los jefes de recursos humanos, directivos y personas a cargo de la selección de personal.

El estudio se justifica debido a que, en la actualidad, las empresas se han adaptado a nuevos cambios y tendencias mundiales. Estos incluyen aspectos tales como la responsabilidad social y ambiental, las tecnologías de información y comunicación, la popularidad de las redes sociales y aplicaciones gratuitas, y las licencias en red, que permiten compartir casi todo. Dentro de estos cambios están también las infinitas opciones de información y de creación que se sirven en bandeja de plata y que están discriminadas de acuerdo con perfiles específicos, en relación con las pesquisas que cada individuo realiza a través de motores de búsqueda como Google. Todo esto les permite a las empresas expandir sus mercados y posicionarse en nichos particulares con estrategias específicas, lo que facilita no solo una producción, una distribución y una comercialización tanto de bienes como de servicios en sectores geográficos que los demandan, sino también ganar oportunidades para su crecimiento empresarial.

De igual manera, las organizaciones actuales deben estar constantemente atentas a la celeridad con la que el mundo global gira, máxime porque esto ha traído consigo cambios culturales que determinan nuevas formas de ver el mundo, una volubilidad de la economía, la existencia de fronteras difusas que hacen efímero y volátil lo que otrora fue exitoso y, con esto, segmentos de mercado cada vez más específicos y exigentes. No en vano, Marshall McLuhan habló del fenómeno de "aldea global" que como profecía se ha cumplido: se han hecho difusas las fronteras de la información y la comunicación, lo que ha virtualizado los espacios y las relaciones, el concepto de público y privado, e incluso una imprecisa identidad de los pueblos.

Por otra parte, debe decirse que esta investigación también se justifica por la necesidad que tienen los programas profesionales de cumplir con altos indicadores de calidad exigidos por el Ministerio de Educación Nacional y el Consejo Nacional de Acreditación (CNA). Este último orienta los lineamientos de alta calidad de la educación superior en correspondencia con los requerimientos internacionales, para que haya una movilidad de las comunidades académicas que realizan 
actividades sustantivas, además de una homologación y una convalidación de los títulos en las universidades extranjeras.

Los resultados dependen de la identificación y descripción de los perfiles de los programas profesionales de Mercadeo, Publicidad y disciplinas afines; del reconocimiento de las tendencias de conocimiento científico y tecnológico de estos campos, y de la categorización de los cargos y funciones requeridos por las empresas y un análisis comparativo entre lo que se oferta desde las comunidades académicas y lo que se requiere en el ámbito laboral colombiano. Las conclusiones de este trabajo de investigación apuntan a que hay un acercamiento entre lo que ofrece la academia y las necesidades empresariales de todos los sectores económicos del país. Finalmente, se debe decir que los resultados del estudio pueden tomarse como un punto de partida para el inicio de otras investigaciones que pueden aportar a las comunidades académicas y gremiales para la consecución permanente de mejores indicadores de calidad en la educación superior.

\section{Problema de investigación}

Actualmente, existen en Colombia 28 programas de Mercadeo y disciplinas afines asociadas al Aspromer. Esta asociación está interesada en ofrecer información confiable que sirva para la toma de decisiones pedagógicas y curriculares para el avance de los programas de acuerdo con las demandas, no solo empresariales, sino también propias de la normatividad vigente del Ministerio de Educación Nacional y del CNA, quien rige, controla y vigila el servicio de educación superior en el ámbito nacional.

El reconocimiento de las oportunidades de desempeño es una de las preocupaciones fundamentales de los programas que ofrecen estas disciplinas, dado que de esto depende tanto su posicionamiento como el reconocimiento académico, gremial y social de sus egresados. Mantener en vigencia los programas depende en gran medida de seguir las tendencias en el conocimiento científico y tecnológico, además de estar al tanto de las necesidades ocupacionales del medio empresarial.

Se entiende entonces que las comunidades académicas deben adaptar constantemente los currículos a las dinámicas científicas, tecnológicas y empresariales, desde lo local, lo regional, lo nacional y lo internacional, para formar a un profesional pertinente y competitivo que dé respuesta a las necesidades empresariales del mundo globalizado.

La definición de las nuevas oportunidades de desempeño de un profesional en Mercadeo, Publicidad y disciplinas afines abre un horizonte de posibilidades a las empresas, que de alguna forma solo tienen una mirada tradicional sobre las funciones de este experto. También ayuda que haya una especialización en las áreas de desempeño de estos profesionales. Esto hace que se cualifiquen los procesos al interior de las organizaciones y se responda a las condiciones económicas actuales del país derivadas de la firma de diferentes tratados de libre comercio con Estados Unidos, Corea y Canadá, respectivamente. Esto genera la adaptación a esta nueva condición de mercado y representa un reto, por cuanto exige nuevas formas de competir en un mercado diverso, de captar nuevos clientes y de buscar futuras inversiones y alianzas.

Es pertinente indagar sobre los ámbitos que corresponde a las nuevas oportunidades de desempeño de los profesionales de Mercadeo o Publicidad, pues a partir de esto se puede llegar a establecer con confiabilidad las oportunidades de desempeño apropiadas para el contexto laboral. También se permite que las facultades que ofertan estos programas académicos sean flexibles en su adaptación a estas tendencias y, por ende, estén abiertas a los cambios latentes en los espacios de práctica real. Así un estudiante puede encontrar caminos diversos al finalizar su carrera o, si se precisa, antes de hacerlo; también puede ver que lo ofertado en el pénsum de su carrera responde a las divergencias en el mercado, ofrece alternativas para crear nuevas empresas $y$, asimismo, le permite ser crítico y reflexivo frente a una oportunidad o a una amenaza.

\section{Antecedentes}

En este apartado se presentan la evolución y las definiciones de los conceptos propios de este estudio, aportadas por autores que también están interesados en el tema.

Laime y Del Valle sostienen que hoy en día las instituciones de educación superior se encuentran enfrentadas al reto de adecuar y actualizar los contenidos curriculares y los títulos ofrecidos a los nuevos perfiles profesionales, surgidos como consecuencia de las transformaciones acontecidas tanto en el mundo productivo como en la nueva realidad del empleo. En esta investigación se buscó determinar las tendencias 
en el diseño de los perfiles profesionales y caracterizarlos. Para esto, se utilizó la técnica de investigación documental que analiza y compara diversas fuentes de información. Los datos primarios se obtuvieron de universidades y de organizaciones internacionales, que han realizado estudios al respecto. Una sólida formación básica, el desarrollo de una capacidad para aprender en el mismo ambiente de trabajo y el cultivo de valores éticos son la garantía de que el perfil profesional será el que mantendrá una visión humana integral significativa a lo largo de todo el diseño curricular.

Es importante destacar que también Hawez y Corvalán afirman que el diseño de las profesiones ha sido una práctica habitual y constante en las universidades y otros centros de formación. En este proceso, un concepto utilizado con frecuencia, de manera casi constante, es el de "perfil profesional". Sin embargo, las diferentes propuestas que se hacen al público -que finalmente es el "consumidor" de este tipo de producto- permiten visualizar cómo las descripciones son genéricas, ambiguas y sumamente convergentes, además de decir poco acerca de qué es lo que realmente es capaz de hacer un egresado. De igual manera, las nuevas tendencias del marketing son una serie de técnicas o estrategias innovadoras, de las cuales no hay mucha información ni muchos estudios.

Según Hernández, Correal-Gaviria y SandovalVargas, existen estudios que han permitido descubrir nuevas formas del marketing que tienen en cuenta más a fondo al cliente y lo hacen experimentar de una manera más cercana un contacto con el producto.

No se puede dejar de lado que la escena actual muestra que la exigencia hacia los profesionales ha cambiado. Estamos en un contexto donde se hace necesario responder a demandas más específicas. Esto hace que los profesionales tengan que formarse constantemente para dar respuesta a problemas con mayor nivel de complejidad. Este fenómeno está enmarcado en condiciones que han ido implementándose como consecuencia de la globalización, la explosión demográfica, la variación económica constante, el subdesarrollo, el cambio cultural de las sociedades, la guerra, la contaminación, las epidemias, solo por mencionar algunos de estos elementos (Martínez-López, LópezJaramillo y López-García).

Del mismo modo, García, Ortoll y Cobarsi, a partir del análisis de ofertas de trabajo, detectan las necesidades del mercado laboral español actual en aquellos aspectos vinculados con la documentación, la gestión de la información y la gestión del conocimiento. Se evalúa si estos perfiles y funciones quedan total, parcial o escasamente cubiertos por la actual titulación en Información y Documentación. Este trabajo constituye una aproximación a las tendencias del mercado e invita a evaluar hasta qué punto los planes de estudio diseñados en el marco del EEEs y el mismo Libro Blanco del Título de Grado en Información y Documentación satisfacen las tendencias y necesidades del mercado laboral. Por último, se mencionan carencias formativas detectadas en el Libro Blanco para cubrir algunas de las ofertas de trabajo, y se proponen líneas de investigación para concretar más el significado del concepto "profesional de la información".

Ahora bien, para dar sentido a la construcción de un perfil profesional con base en las demandas de las organizaciones, así como para discriminar los perfiles profesionales de los 28 programas académicos de Mercadeo y disciplinas afines que hay en el país, es importante precisar el concepto de profesión. Cabe señalar que este ha estado ligado a la evolución y al desarrollo de las sociedades. Esto hace un poco difícil tener una definición única de qué es una profesión, puesto que existe una frontera difusa entre lo que es una ocupación y una profesión (Fernández). Sin embargo, es posible identificar algunos elementos, como la formación educativa de alto nivel, la estructura organizativa, la vinculación con las estructuras de poder, el estatus, la actualización continua y la búsqueda de autonomía; estos factores hacen que una actividad determinada sea considerada una profesión.

Por otra parte, la competencia ha de identificar aquello que necesita cualquier persona para solucionar los problemas con los que se enfrentará a lo largo de su vida. Por lo tanto, esta consistirá en la intervención eficaz en los diferentes ámbitos de la vida mediante acciones en las que se movilizan, al mismo tiempo y de manera interrelacionada, componentes actitudinales, procedimentales y conceptuales.

- Estas son acciones eficaces ante situaciones y problemas de distinto tipo que obligan a utilizar los recursos de que se dispone.

- Para dar respuesta a los problemas que plantean estas situaciones, es necesario estar dispuestos a resolverlos con una intención definida, o sea, con unas actitudes determinadas.

- Una vez mostrada la disposición y el sentido para resolver los problemas planteados con unas actitudes determinadas, es necesario dominar los procedimientos, habilidades y destrezas para la acción que se debe llevar a cabo. 
- Pero, para que estas habilidades lleguen a buen fin, deben realizarse sobre unos objetos de conocimiento, es decir, estar basadas en unos hechos, unos conceptos y unos sistemas conceptuales. Todo esto ha de realizarse de forma interrelacionada: la acción implica una integración de actitudes, procedimientos y conocimientos (Zabala y Arnua).

La competencia se da entre el mundo de los saberes y las habilidades que se han desarrollado a través del tiempo. Nunca viaja sola: está siempre acompañada de la acción y jamás puede darse sin conocimiento. Se concibe pues como un conjunto de potencialidades que se reinventa y modifica con el fin de responder situaciones específicas o crisis organizacionales que ameriten incluso un cambio de paradigma (Gallard y Jacinto).

Aunado con lo anterior, la competencia profesional y laboral involucra un cúmulo de experiencias significativas y útiles que, sin estar estrictamente ligadas a la academia, han de ser el sustrato para alcanzar un exitoso desempeño profesional.

A estos dos conceptos se suma uno muy citado en este artículo, el de "perfil profesional", y este se subdivide en categorías. Primero está el profesional básico, quien es el recién egresado que asume el cumplimiento eficiente de tareas para las cuales fue formado en el alma máter. Otra categoría es la de "profesional experto", quien es el trabajador que, luego de recibir el diploma, ha adquirido experiencia en una o más organizaciones y que, junto con la formación que recibió en el pregrado, ha sumado la experiencia propia del día a día en un entorno real, enfrentándose a los cambios del mercado, a las crisis, a las disposiciones gubernamentales, entre otras. Le sigue el profesional especialista, quien ha decidido sumar títulos adicionales al del pregrado y que adicional tiene experiencia laboral.

Otro concepto importante es el de "mercadotecnia", pero esta vez entendido con un enfoque social, y está acorde con las nuevas formas de ver la evolución de las comunidades y el futuro de la humanidad. En la actualidad este tipo de actividad no está solo pensada en un ámbito lucrativo; por el contrario, se enfoca en el desarrollo sostenible y en proponer alternativas viables que garanticen la existencia de los seres humanos y la supervivencia del planeta.

Lo anteriormente expuesto permite una mejor comprensión del tema aquí tratado. También abre la posibilidad de continuar con el debate que estos conceptos sugieren debido al constante cambio desde la académico, científico y tecnológico.

\section{Metodología}

Este tipo de estudio es descriptivo con un enfoque cuantitativo y con un muestreo aleatorio simple, en razón de las características de las fuentes de información primarias, además porque este tipo de estudio da mayor validez a los datos recogidos. El análisis de la información recogida a través de instrumentos, como encuestas y documentos, permitió identificar las nuevas oportunidades de desempeño laboral de los profesionales de Mercadeo y Publicidad, teniendo en cuenta además las tendencias científicas, tecnológicas y empresariales para la reflexión y toma de decisiones pedagógicas y curriculares en los programas asociados a Aspromer.

Para llevar a cabo el estudio, se realizó una descripción tanto de los perfiles profesionales como de los ocupacionales de los programas de Mercadeo y Publicidad en Colombia, se establecieron las necesidades empresariales en referencia al desempeño de los profesionales en esas áreas y finalmente se recomienda seguir los resultados del estudio para que los perfiles profesionales actuales estén en sintonía con las nuevas tendencias de desempeño laboral de los profesionales y respondan a las necesidades del medio empresarial globalizado.

\section{Definición de los sujetos de estudio}

Se tuvieron en cuenta los 28 programas de Mercadeo, Publicidad y disciplinas afines que existen en el país que estuvieran inscritos en Aspromer hacia febrero del 2014, los cuales aportaron la información documental sobre los perfiles profesionales. Por otra parte, se consideraron 136 grandes y medianas empresas inscritas a la Cámara de Comercio de las cinco ciudades principales de Colombia (Bogotá, Medellín, Cali, Barranquilla y Bucaramanga); estas aportaron información mediante una encuesta, cuyo análisis permitió establecer las necesidades empresariales en referencia al desempeño de los profesionales de Mercadeo y Publicidad. Esta encuesta fue aplicada a los jefes de recursos humanos, a los directivos y a las personas a cargo de la selección de personal. Cabe destacar que las encuestas se llevaron a cabo a través del correo electrónico, y se realizó un seguimiento telefónico. La muestra para la aplicación 
del instrumento fue aleatoria simple con un nivel de confianza del $90 \%$. Inicialmente se desarrolló un cuestionario con preguntas de selección múltiple, en escala tipo Likert, y algunas preguntas abiertas. Para validar el instrumento, se realizó una prueba piloto para asegurar que esta midiera con rigor las variables seleccionadas. La encuesta estuvo construida por 10 preguntas, que estaban distribuidas de la siguiente manera: ocho preguntas eran de selección múltiple, una pregunta estaba en escala de Likert y dos preguntas eran abiertas, las cuales permitieron reconocer los cargos y funciones de los profesionales en mención. La estrategia de aplicación se realizó a través de la plataforma en línea Encuesta Fácil.

La metodología se estructuró con base en tres objetivos específicos. El primero buscaba identificar los perfiles profesionales y ocupacionales de los programas de Mercadeo, Publicidad y afines en Colombia, para lo cual se realizaron las actividades anteriormente mencionadas, lo que permitió condensar información valiosa respecto a los perfiles profesionales que se ofertan en los programas de pregrado. El segundo objetivo consistió en describir las tendencias científicas y tecnológicas de Mercadeo, Publicidad y disciplinas afines, lo que supuso una revisión teórica de la bibliografía de estas áreas de conocimiento, así como hacer el estado del arte de estas disciplinas desde el presente y con una visión orientada hacia el futuro. Los principales hallazgos del 2012 indican que en un mundo marcado por las redes sociales debe existir una reingeniería en el uso de medios. Debe tomarse en cuenta la eficiencia, crear modelos híbridos de comunicación, se deben a su vez, abrir nuevos caminos de interacción e interactividad y pensar en red.

Así mismo, tiene mucha más fuerza el medio que el contenido que se transmite, máxime porque el publicista o el experto en mercadeo está enfrentado a una era cuando las tabletas y los teléfonos inteligentes están a la orden del día, y tienen toda una oferta de posibilidades para captar en multimedia a los usuarios y lograr que sean ellos mismos los que hagan viral el mensaje.

En Mercadeo, en el 2012, se habló de las tendencias del targeting, específicamente del behavioural targeting y redes sociales, el cual aprovecha los perfiles de los usuarios para conocer sus gustos y deseos, lo que permite que se segmenten mucho más las ofertas. También está el geotargeting y móviles, el cual se sirve del uso que le dan las personas a Internet fuera del hogar, particularmente desde teléfonos inteligentes y otro tipo de dispositivos, lo que ayuda a los clientes a enlazarse a través de campañas móviles de crossmedia. A esto se suman otras tendencias más, y vale la pena particularizar el canal de YouTube que personaliza el tipo de videos de acuerdo con las visitas del usuario.

Asimismo, para el 2013, se planteó una mayor segmentación del target de forma simple y sencilla con un mensaje individualizado en concordancia con los gustos y las búsquedas que un individuo realice. Se habla de un entorno mucho más audiovisual donde prima el video como forma de contar y de interrelacionarse.

Para el 2015, la tendencia más fuerte es la de pagos a la distancia de un clic. Asimismo, hay marcas que patrocinan totalmente guiones de películas o seriados de televisión.

De igual forma, existen aplicaciones y se están desarrollando muchas más para dispositivos móviles, con modelos más flexibles y con tecnologías que se enfocan en la productividad y facilitan la movilidad de los trabajadores en las empresas, lo que hace real el concepto de "oficina virtual" y una nueva forma de relacionarse.

Cabe anotar que estas son solo algunas de las tendencias que marcaron y definirán mucho del panorama al que deben enfrentarse las empresas, así como los profesionales en Mercadeo y Publicidad. No obstante, la gran mayoría de estas se asocian con la era digital y con una sociedad mediatizada en la que la información fluye por múltiples vías; se hace muy difícil definir un tipo de cliente o de mercado de forma estricta, puesto que cada individuo representa una identidad comercial y unos intereses de compra particulares que hacen más compleja la segmentación.

Por último, se hizo una comparación entre las necesidades laborales empresariales de los profesionales de Mercadeo, de Publicidad y de áreas afines y las tendencias científicas de Mercadeo y Publicidad. Para cumplir con este objetivo, se realizó un análisis de las necesidades de las empresas en relación con las tendencias del conocimiento científico y tecnológico, específicamente en el Mercadeo, la Publicidad y campos afines.

De igual manera, se elaboró un balance entre las funciones y cargos de los empresarios; las tendencias de las disciplinas y la definición de los nuevos perfiles, y, por último, una definición de nuevos perfiles de acuerdo con las tendencias de desempeño laboral de los profesionales de Mercadeo o Publicidad. 


\section{Procedimientos}

La búsqueda y análisis de información se realizaron a través de las siguientes técnicas de recolección de datos:

- Se hizo un análisis de fuentes secundarias, que fueron los proyectos educativos de cada uno de los programas de Mercadeo y Publicidad adscritos a Aspromer.

- Se elaboró una revisión documental sobre los avances científicos y tecnológicos en el Mercadeo y la Publicidad

- Para establecer las necesidades empresariales en el desempeño de los profesionales en Mercadeo y Publicidad se utilizó como instrumento una encuesta.

\section{Análisis de los resultados}

Las figuras 1 y 2 muestran la información recolectada a través de la encuesta realizada:

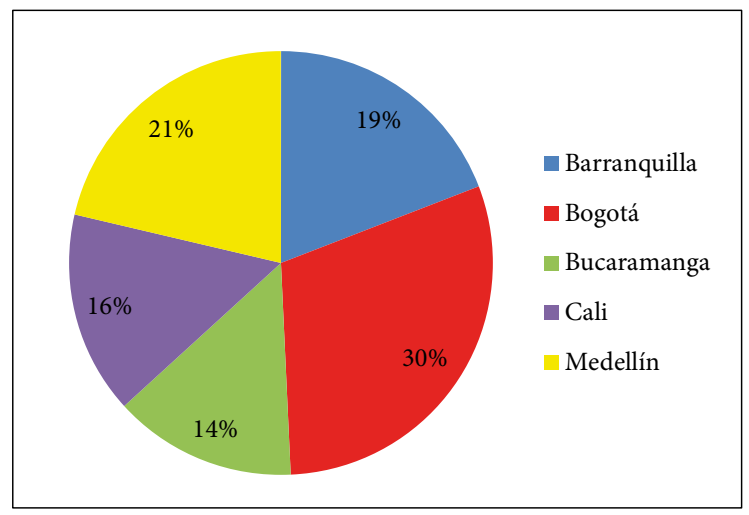

Figura 1. Encuestas realizadas por ciudad.

Fuente: elaboración propia.

En primera instancia, se destaca el número de encuestas realizadas según la distribución por ciudades. Es así que en la capital del país se realizaron 41 encuestas; en Medellín, 29; en Barranquilla, 26, en Cali, 21, y Bucaramanga, 19.

Es importante recordar que las encuestas fueron realizadas a empresas grandes y medianas en sectores económicos que van del primario al terciario. Pero la mayoría de estas (59) pertenecían al sector terciario, en otras palabras, el $49 \%$ de las compañías se encontraban ubicadas en esta sección de la economía.

Otras generalidades de la encuesta son que las empresas pertenecían al sector de comercialización de productos industriales, la industria, la construcción, salud, la educación, el sector financiero, energético, finca raíz, de minerales, entre otros.

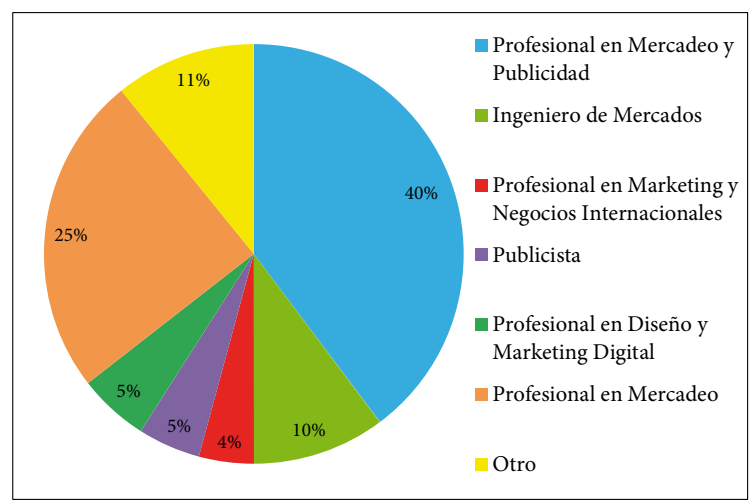

Figura 2. Título del aspirante a trabajar en el área de Mercadeo. Fuente: elaboración propia.

Según la muestra analizada, los encuestados consideran que el título debería describir el tipo profesional que va a trabajar en el área de Mercadeo de su compañía en relación con la ciudad. Estos son "profesional en Mercadeo y Publicidad" con el $40 \%$ de preferencia de los encuestados; un "profesional en Mercadeo" con el $25 \%$; "ingeniero de Mercados" con un 10\%; "profesional en Diseño y Marketing Digital" con el 5\%; publicista también con $5 \%$, y el $4 \%$ sugiere "profesional en Marketing y Negocios Internacionales".

El $21 \%$ considera que el profesional debe ser creativo; el 20\%, manejar estrategias comerciales y de ventas; el 20\%, un planeador estratégico, y el 19\%, investigador de mercados.

En la figura 3 se muestran los profesionales con vínculos laborales en cada una de las empresas.

El $40 \%$ de los profesionales está en el área de gerencia comercial y el $30 \%$, en gerencia de mercadeo; estos dos son los grandes grupos de personas empleadas actualmente en la parte gerencial.

En la parte comercial y de ventas, el $41 \%$ está en la dirección comercial; $19 \%$ es jefe de departamento, jefe de ventas o gerente de ventas; el $11 \%$ es ejecutivo de cuentas clave.

En la categoría de marketing, el $21 \%$ está en dirección de marketing; otro $21 \%$, en investigación de mercados; el $17 \%$, en planeación estratégica; el $10 \%$ es jefe de departamento o jefe de producto; el $8 \%$ director de merchandising; otro $8 \%$ es gerente de marca o de producto; el 6\%, director de trade marketing; el $5 \%$, director de operaciones, y el 3\%, director de relaciones públicas. 


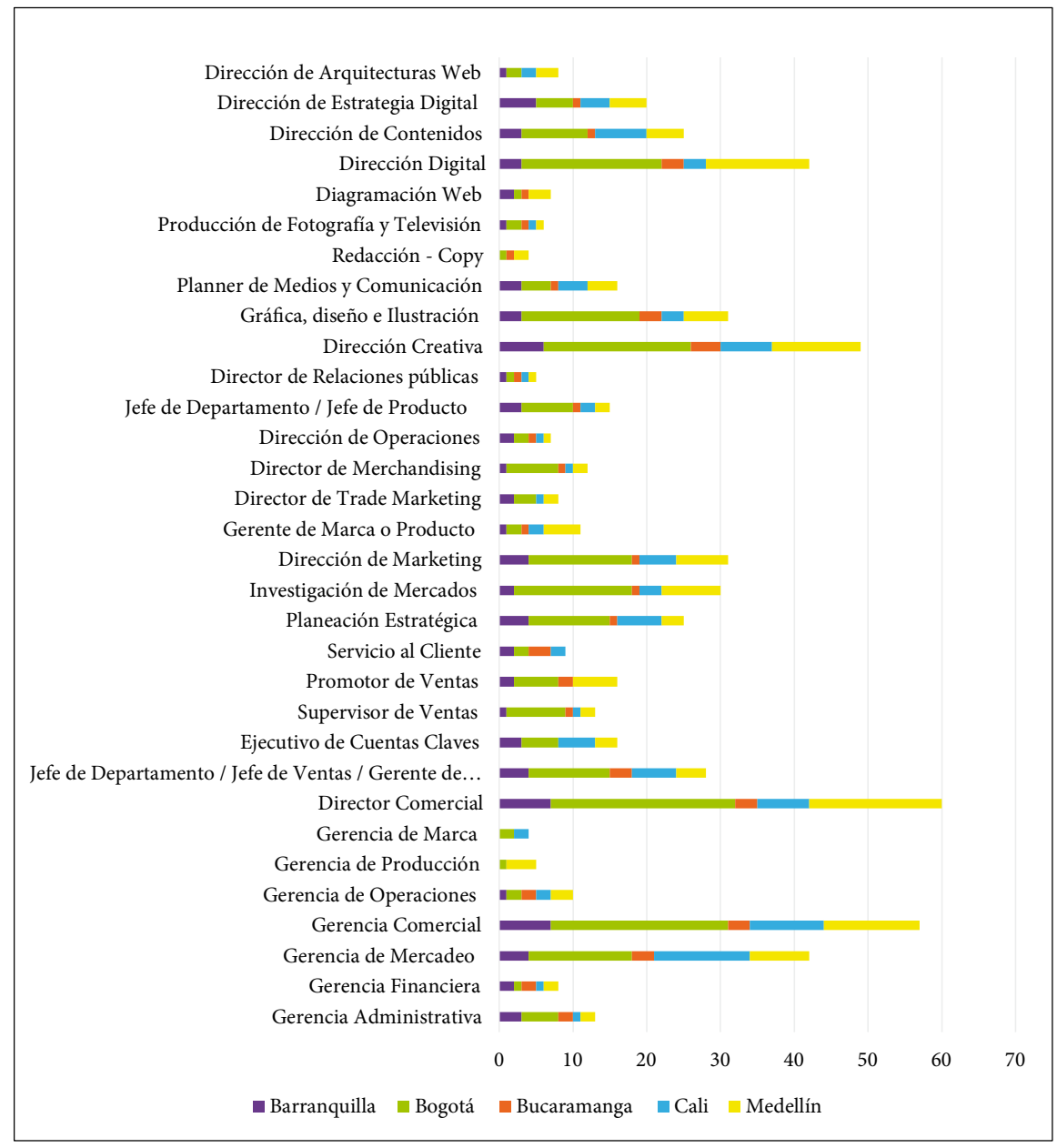

Figura 3. Profesionales en las empresas respecto a cada ciudad. Fuente: elaboración propia.

En publicidad, existe un $41 \%$ de personas empleadas en el área de dirección creativa; el 26\%, en gráfica, diseño e ilustración; el 13\% es planner de medios y comunicación; el 6\% es diagramación web, y $6 \%$ representa otro que, para este caso, son las diferentes personas o agencias externas que manejan dichas cuentas.

En tecnología, informática y medios digitales, un $43 \%$ está en dirección digital, un $26 \%$, en dirección de contenidos; $21 \%$, en dirección de estrategia digital, y el $8 \%$ está en dirección de arquitectura web.

Se analizó también en qué ciudad se encuentran mayoritariamente los profesionales en dirección digital, y se concluyó que la mayoría de personas en ese cargo está en Bogotá y Medellín. Lo mismo ocurre para la labor de dirección creativa y dirección comercial. En gerencia de mercadeo, las ciudades son Bogotá y Cali.

Se encontró que las habilidades que debe tener un profesional son, destacando como la más importante, la iniciativa, con el 13\%; seguida por trabajo en equipo y facilidad de comunicación con el $12 \%$, respectivamente; trabajo bajo presión, creatividad y liderazgo con el 9\%; recursividad con el 7\%; autoconfianza con el 6\%; capacidad de negociación con el 12\%; flexibilidad y tolerancia a la frustración con el 5\%, correspondientemente, y finalmente recursividad y capacidad de organización con un $4 \%$ cada una. 


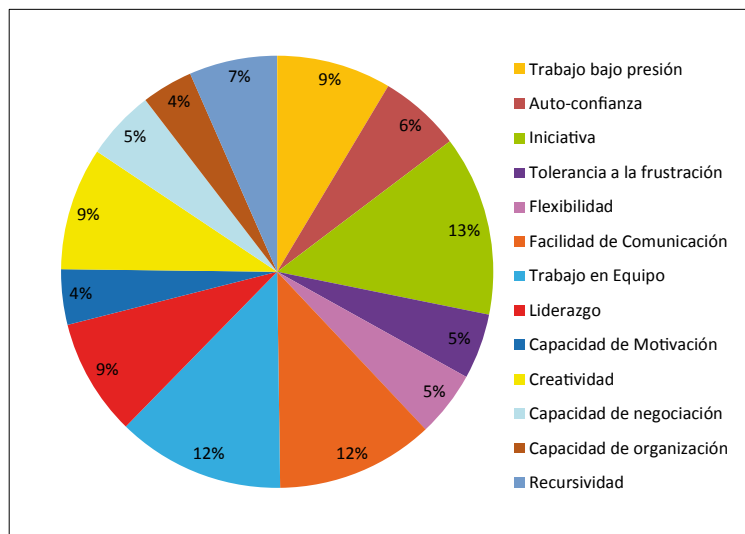

Figura 4. Habilidades para un profesional en Mercadeo y Publicidad. Fuente: elaboración propia.

Partiendo de estos datos, se puede inferir que las empresas buscan, además de la formación, que el profesional aporte nuevas ideas y no se limite solo a hacer lo que se encuentra en su manual de funciones. De esta manera, puede decirse que la recursividad es un rasgo importante del profesional, es decir, ser capaz de resolver situaciones en medio de una crisis o de ser asertivo en un momento coyuntural. Aunado con esto, los requerimientos empresariales precisan un trabajador que sea capaz de integrarse positivamente al equipo de trabajo y que tenga habilidades comunicativas tanto en orales como escritas (figura 5).

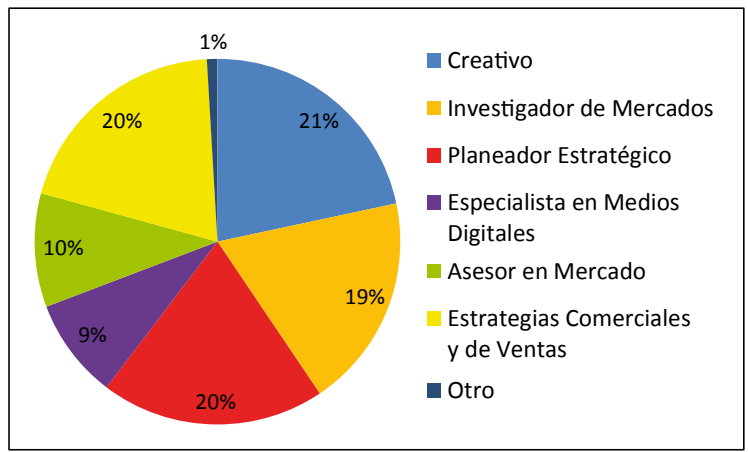

Figura 5. Perfiles y áreas del profesional.

Fuente: elaboración propia.

La torta da cuenta de que una de las principales cualidades que se busca en un profesional es la creatividad. El 21\% de los encuestados lo reafirma. Con un porcentaje similar, un $20 \%$, se espera que este cree, planee o aplique estrategias comerciales y de ventas. Pero es preciso tomar en cuenta otros aspectos de la figura. Por ejemplo, en las empresas se busca que un profesional se especialice en medios digitales.
Esto puede deberse a que las organizaciones son cada vez más conscientes de la apertura del mercado y de la necesidad de servirse de los medios digitales para proyectar sus productos y servicios. En la actualidad, lo audiovisual y lo sonoro cobran mucha importancia, y más aún si se comparten en las plataformas web o en redes como Facebook, YouTube, Instagram, etc. Asimismo, se buscan perfiles de asesoramiento en temas de mercado y estrategias comerciales y de ventas con el $10 \%$ y el $20 \%$, respectivamente.

Los campos en que se puede desempeñar con mayor efectividad cada profesional de acuerdo con la valoración de cada uno de los encuestados son los siguientes. Primero, se pueden desempeñar en el campo de la investigación de mercados; se considera que los profesionales en Mercadeo y en Mercadeo y Publicidad son los más competentes, seguidos de los ingenieros de Mercados. En el área de ventas, encontramos a los profesionales en Mercadeo y Publicidad, a los profesionales en Mercadeo y a los ingenieros de Mercados. En trade marketing aparecen en escena los profesionales en Mercadeo y Publicidad, los profesionales en Mercadeo y, muy minoritariamente a los ingenieros de Mercados. En todos los campos de desempeño se encontró que el más competente, en relación con otros títulos, es el profesional en Mercadeo y Publicidad, pues la mayoría de las empresas entrevistadas - 66, para ser más precisos- manifestó que el título que debe tener un profesional que trabaje en el área de Mercadeo debe ser el de profesional en Mercadeo y Publicidad. No obstante, 41 organizaciones encuestadas dijeron que en este podía estar un profesional en Mercadeo; 17 afirmaron que un ingeniero de mercados; nueve, que un profesional de Diseño y Marketing Digital; ocho, que un publicista; siete, que profesionales en Marketing y Negocios Internacionales, y 18, un título alterno, que podía ser ingeniero industrial o civil con alguna especialización en temas de Mercado, un administrador de empresas, etc.

Así mismo se realizó una pregunta abierta: ¿en qué campo se puede desempeñar un profesional de Mercadeo y Publicidad en su empresa? Esto para determinar con exactitud las áreas en las que un profesional de Mercadeo y Publicidad puede trabajar. Es importante precisar que esta no era una pregunta obligada, por lo cual solo 79 empresas respondieron. Los resultados fueron variados. Hubo 28 alternativas entre las que se cuentan: gerente de Mercadeo, áreas administrativa, comercial y ventas, mercadeo, desarrollo de nuevos mercados, atracción de nuevos clientes, branding, merchandising, contacto directo con el 
cliente externo, entre otras. Pero se destaca que las áreas comercial y de ventas tuvieron 28 respuestas con el 35\%; le siguió mercadeo con 17 y un $21 \%$; publicidad, con 8 , lo que porcentualmente significa $10 \%$.

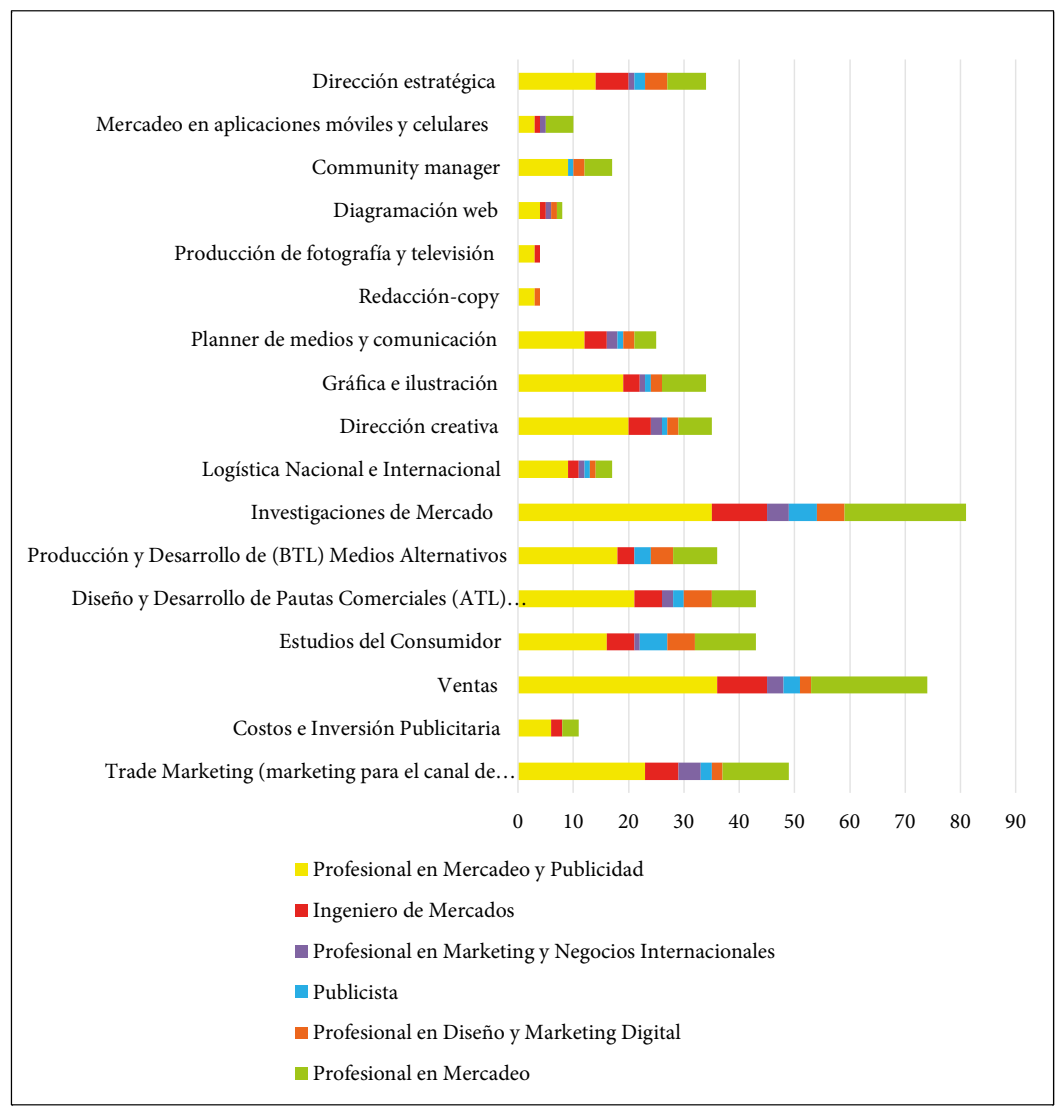

Figura 6. Campo de desempeño laboral de acuerdo con el título profesional. Fuente: elaboración propia.

No obstante, de acuerdo con los nuevos avances tecnológicos y científicos, hay nuevos campos que para el Mercadeo y la Publicidad, entre los que se cuentan la investigación y desarrollo; el mercado y la circulación; la dependencia de calidad; el marketing electrónico; ser community manager; ser un shopper, etc. Por esto, se consultó a las 136 empresas sobre qué nuevos cargos en relación con los avances en materia de ciencia y tecnología les eran más conocidos. Entre los datos obtenidos se aprecia lo siguiente: reconocen el de community manager, el de trade marketing y el mercadeo en dispositivos móviles; se menciona también el CMR digital.

Respecto al mercadeo, hay términos que son desconocidos por parte de los encuestados. La figura 7 muestra los resultados que arrojó la encuesta.

De la figura 7 se deduce que, de las 14 tácticas usadas en el Mercadeo, las más conocidas son la investigación de mercados, el brandmanagement y los canales de distribución, y las menos conocidas, el coolhunting (la cazatendencias), la minería de datos, el brandequity, el key account.

También se observa que el trade marketing no es conocida con 50; los canales de distribución son bien apreciados con 43, las estrategias push/pull of products no son famosos con 49; el branding es poco notado con 41; el brandmanagement (la gestión de la marca) no es conocida con 46; la brandequity tampoco lo es con 57; el coolhunting tampoco con 75; la minería de datos y el marketing interactivo no son conocidos con 47; el neuromarketing no es conocido con 50; el key account no es conocida con 59; la investigación de mercados es bien conocida con 51, la labor de community manager es poco conocida con 50 , y el network marketing no es conocido con 50. 


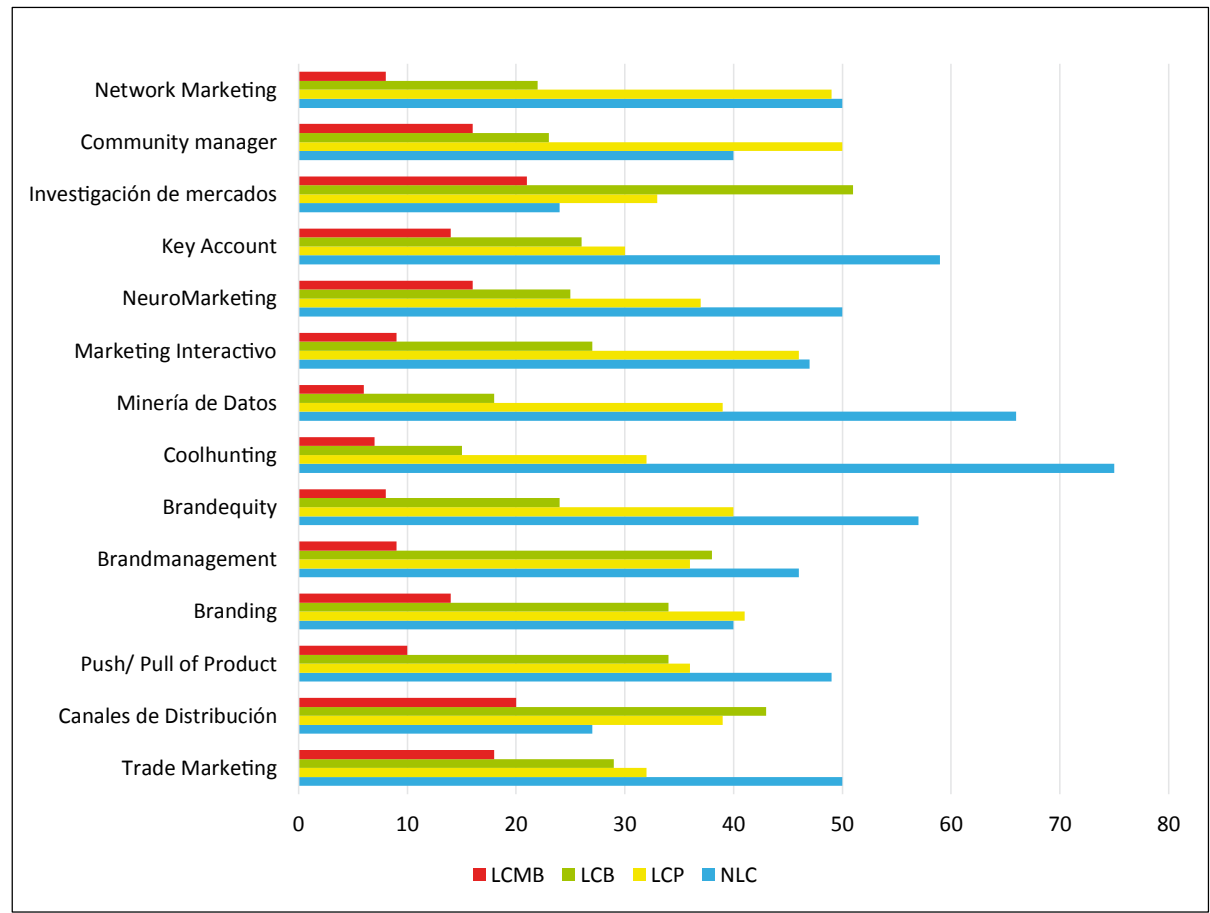

Figura 7. Niveles de conocimiento de tácticas en mercadeo.

Fuente: elaboración propia.

\section{Conclusiones}

Es importante resaltar que este tipo de estudios, y el de "Tendencias de la Oferta Laboral Colombiana de Profesionales en Mercadeo y Publicidad", son necesarios porque abren un abanico de posibilidades en la oferta de campos de acción para un profesional en Mercadeo y Publicidad. Se habla de la oferta porque los cambios tecnológicos y científicos, además de que el mundo esté totalmente globalizado y permeado por Internet, hacen que un estudiante que esté ad portas de obtener su título profesional o un recién egresado tengan una mirada mucho más plural sobre su quehacer y su enfoque laboral. Si bien esto significa que el medio se hace más exigente y es necesario que haya mucha más investigación y conocimiento del mercado, como también de que sea dinámico y voluble, lo cierto es que hay muchas formas más de hacer llegar la información que solo a través de medios tradicionales, como la radio, la prensa o la televisión, y que, asimismo, son variados los niveles de especialización para un mismo cargo. Entre estos se destacan el community manager o social media manager, quienes deben estar siempre al tanto de lo que ocurre en el ámbito digital entre la empresa, la marca y sus clientes. Conocer lo que ellos ven, escuchan y publican en redes sociales como Facebook, Twitter, YouTube, etc., así como los comentarios o notificaciones en blogs, memes o quotes, es clave para desarrollar contenidos creativos, estrategias, conocer tendencias y pensar soluciones para el desarrollo de productos y la mejora de procesos.

El perfil de un egresado en el área de Mercadeo y Publicidad es el de alguien dinámico, abierto al cambio y consciente de que debe especializarse. También debe tener conocimientos sobre todas las tendencias y las propuestas de desempeño laboral que en la actualidad ofrece el mercado. Es claro que los empresarios prefieren una mezcla entre títulos, como un profesional en Mercadeo y Publicidad (40\%), un profesional en Mercadeo (25\%), un ingeniero de Mercados (10\%), un profesional en Diseño y Marketing Digital (5\%) y un publicista (5\%). Esto implica que debe estar abierto respecto a nuevos aprendizajes y que esté en constante actualización.

Un nuevo profesional en Mercadeo y Publicidad debe estar dispuesto a gerenciar proyectos y tener una amplia visión comercial en relación con las nuevas tendencias de marketing, los cambios económicos, así como de los tratados de libre comercio firmados 
con Estados Unidos, China y Canadá, que dan otra mirada al mercado local y ponen a prueba estrategias de posicionamiento de marca y desarrollo de productos que el cliente desee.

Los nuevos profesionales deben amar la tecnología, deben tener como cualidad particular el pensamiento creativo y, de manera simultánea, el pensamiento estratégico. En suma, deben ser artistas y geeks.

A raíz de estos mismos avances, un profesional en Mercadeo y Publicidad ha de buscar alternativas en nuevos medios, puesto que, en la actualidad, las personas consumen desde diferentes dispositivos y plataformas como los teléfonos móviles, las tabletas, entre otros. Deben buscar espacios en los cuales prime mucho lo visual, así como los mensajes concretos que apuntan no a un nicho de mercado, sino a personas con cualidades y gustos individuales y específicos. El individuo tiende a buscar cosas muy particulares.

Las personas actualmente son muy del concepto remix, un anglicismo que designa mezclas en la música, pero que bien aplica a los gustos estéticos en todo sentido: tienen una visión mucho más cosmopolita y global de la cultura, y las artes se abren como un abanico de opciones. Asimismo, más que vender un producto, las empresas, en la actualidad, deben orientarse al posicionamiento de una marca y una idea desde las áreas de Mercadeo y Publicidad. En la publicidad cuenta mucho el mensaje y el modo cómo este llega a los usuarios. Las personas buscan empresas comprometidas con el planeta, que tengan responsabilidad social. Deben tener prioridad los mensajes que hablen de la amistad, de la convivencia, del respeto por los animales, que apunten al humor, más que al consumo o a la adherencia fiel a un producto, bien o servicio.

Se encontró que un $40 \%$ de los empresarios prefiere un profesional en Mercadeo y Publicidad; el 25\%, un profesional en Mercadeo, y un 10\%, un ingeniero de Mercados. Se considera que su preferencia por el profesional en Mercadeo y Publicidad se debe a que este maneja dos disciplinas muy afines y complementarias.

El 21\% considera que el profesional debe ser creativo; el 20\%, manejar estrategias comerciales y de ventas; junto con el $20 \%$ que lo ve como un planificador estratégico, y el 19\%, como investigador de mercados.

Los perfiles de las instituciones están en concordancia con las identidades corporativas y su plan de estudios, lo que permite deducir que los profesionales de estas disciplinas que se vienen formando en el país son pertinentes no solo para desempeñarse laboralmente en el ámbito interno, sino también en el externo. Las tendencias científicas y tecnológicas corresponden a estos perfiles, lo que muestra que estas tendencias se tienen en cuenta por parte de las comunidades académicas nacionales para fortalecer los perfiles profesionales.

En el análisis comparativo de perfiles, tendencias y necesidades empresariales, se ve la importancia de cada una de las variables estudiadas, porque permiten a las instituciones de educación superior realizar los ajustes en cada perfil profesional teniendo en cuenta el currículo de las profesiones más acordes con el contexto local y global. Es por esto que, aunque los resultados obtenidos se deben considerar como un aporte inicial para la actualización de estos perfiles, no se vio la necesidad de una propuesta nueva, pues las respuestas de los empresarios muestran que hay una correspondencia entre los perfiles que vienen empleando y sus necesidades laborales actuales.

Las empresas expresaron la necesidad de profesionales competitivos en las áreas estudiadas y no se alejan de los perfiles profesionales que se vienen formando. Las instituciones muestran currículos flexibles que dan cuenta que día a día pueden ser ajustados teniendo en cuenta las tendencias científicas y tecnológicas, acorde con las demandas de dichas empresas.

Al hacer la recopilación y el análisis de las tendencias a nivel científico y tecnológico, se pudo ver que no se pueden tomar decisiones estratégicas de forma permanente en un mundo con tácticas que cambian diariamente, los profesionales en estas áreas deben estar a la vanguardia con lo que sucede en estos campos.

De forma conclusiva, las tendencias en el perfil del profesional en Mercadeo y Publicidad, de acuerdo con los avances científicos y tecnológicos en estas disciplinas, y a la consulta, la revisión de artículos y la bibliografía al respecto, son las siguientes:

- En la próxima década, los profesionales en Mercadeo y Publicidad serán artistas y geeks. El pensamiento estratégico y analítico se fusionará con el talento creativo.

- Los profesionales del futuro tendrán que aprender a desenvolverse en múltiples ámbitos. No podrán permitirse el lujo de encerrarse en las áreas en las que se sienten más cómodos. Deberán salir fuera de su departamento para desempeñar muchas veces las tareas de colaborador y de coordinador.

- Si quieren sobrevivir en el futuro, los profesionales en Mercadeo y Publicidad tendrán que ser 
agnósticos sobre la tecnología y los canales. Los cuatro o seis canales publicitarios con que se cuenta hoy en día podrían convertirse en decenas o centenares para el 2020.

- Hoy en día, la mayor parte de los departamentos de marketing están poblados por profesionales en Mercadeo. Para el 2020, los departamentos de marketing estarán dominados por "inmigrantes" del marketing, como los psicólogos cognitivos, los matemáticos, los científicos de datos, los sociólogos y los desarrolladores de software. En el futuro, el departamento de marketing será completamente multidisciplinar.

- De cara a los próximos años, se deberá hacer hincapié en la provisión de servicios y de experiencias relevantes para el cliente, más que en vender un determinado producto.

- Para el 2020, el marketing "para las masas" habrá pasado ya a la historia. El marketing dejará de mirarse en el espejo de los medios de comunicación masivos para hacerlo en el de la segmentación, la personalización y el microtargeting.

- En el futuro, los directores de marketing invertirán más en tecnología que sus propios homólogos del departamento de tecnología. Si quieren subsistir en la nueva era digital, los profesionales en Mercadeo y Publicidad tendrán que aprender a ser líderes tecnológicos.

- Los datos serán el gran apoyo de los profesionales en Mercadeo y Publicidad en los próximos años, pero también pueden convertirse en sus peores enemigos si no se muestran precavidos. Los datos son una mina de oro desde el punto del marketing, pero también entrañan graves amenazas, sobre todo desde el punto de vista de la privacidad, a las cuales los profesionales estarán obligados a reconocer.

- Los días del tradicional plan anual de marketing habrán pasado a la historia en para el 2020. Los profesionales seguirán teniendo un plan anual de trabajo, pero se verán forzados a realizar continuos ajustes sobre este.

- Como los hombres del Renacimiento, los profesionales en Mercadeo y Publicidad del futuro serán multidisciplinares e integrarán conocimientos de marketing, de publicidad, de relaciones públicas, de psicología, de tecnología, de neurociencias, de diseño, de datos, etc. Estos tendrán que aprender a destacarse y a moverse en diferentes ámbitos y salir de sus zonas de confort. Aunado con lo anterior, estos no podrán quedarse solo en frente de su escritorio, sino salir, tener contacto con las personas, coordinar; también ejecutar procesos y actividades

- Dentro de unos años, el mercado será el que defina los mensajes de los profesionales. Por eso, estos deberán buscar de manera constante la retroalimentación y las experiencias del cliente para incorporarlas a estos. El marketing boca a boca, que ha sido siempre una de las piedras angulares de esta disciplina, cobrará aún más importancia en el futuro, ya que las personas creen más en las recomendaciones de terceros y menos en la publicidad de una marca.

\section{Referencias}

Fernández-Pérez, Jorge. "Elementos que consolidan al concepto profesión: notas para su reflexión”. Revista Electrónica de Investigación Educativa 3.1 (2001): 23-39. Web.

Gallart, María Antonia y Claudia Jacinto. Competencias Laborales: tema clave en la articulación educación-trabajo. Curso subregional de formación de gerentes de educación técnico-profesional. 1995. Web. Enero del 2014. <http://www.cinterfor.org.uy/public> .

García, Montserrat, Eva Ortoll y Josep Cobarsi. Tendencias en el mercado laboral: nuevos perfiles profesionales en Información y Documentación. Barcelona: Universitat Oberta de Catalunya, 2009. Impreso.

Hawez, Gustavo y Óscar Corvalán. Construcción de un perfil profesional. Talca: Universidad de Talca, 2006. Impreso.

Hernández-Rincón, Francy, Angélica Correal-Gaviria y Dayan Sandoval-Vargas. Estudio teórico de las nuevas tendencias del marketing. Chía: Universidad de la Sabana, 2006. Impreso.

Laime, Susan y Liliana del Valle. "Tendencias en el diseño de perfiles profesionales". Encuentro Educacional 10.1 (2003): 46-57. Web.

Martínez-Lopez, Julián, Moisés López-Jaramillo y Paulo López-García. Estudio de mercados acerca de las necesidades de educación continuada y perfil ocupacional del egresado de Licenciatura en Educación Física de la Universidad de Antioquia. Medellín: Universidad de Antioquia, 2007. Impreso.

Zabala, Antoni y Laia Arnua. "La enseñanza de las competencias". Revista Aula de Innovación Educativa 161 (2007): 40-46. Web. 